Perceptual \& Motor Skills: Motor Skills \& Ergonomics

2012, 115, 3, 895-902. (C) Perceptual \& Motor Skills 2012

\title{
EFFECTS OF TWO DIFFERENT TRAINING PROGRAMS WITH SAME WORKLOAD ON THROWING VELOCITY BY EXPERIENCED WATER POLO PLAYERS ${ }^{1,2}$
}

\author{
MÁRIO C. MARQUES, SILVÉRIO M. LIBERAL, ALDO M. COSTA \\ Research Centre in Sports, Health, and Human Development \\ University of Beira Interior, Portugal
}

ROLAND VAN DEN TILLAAR

Research Centre in Sports, Health, and Human Development

Nord Trøndelag University College, Norway.
LUIS SÁNCHEZ-MEDINA

Studies, Research, and Sport Medicine Center Government of Navarre, Pamplona, Spain

\section{JÚLIO C. MARTINS AND DANIEL A. MARINHO \\ Research Centre in Sports, Health, and Human Development University of Beira Interior, Portugal}

\begin{abstract}
Summary.-To investigate the effects of two different strength-training programs with the same workload (impulse) on throwing velocity in water polo, 30 water polo players $(M$ age $=17.1 \mathrm{yr}$., $S D=4.9 ; M$ mass $=71.2 \mathrm{~kg}, S D=14.7 ; M$ height $=1.75 \mathrm{~m}, S D=0.09 \mathrm{~m}$ ) were randomly divided in two groups based upon throwing performance with water polo ball. The medicine-ball training group performed $3 \times 6$ reps with a $3-\mathrm{kg}$ medicine ball, while the combination training group completed $1 \times 9$ repetitions with the $3-\mathrm{kg}$ medicine ball, followed by $3 \times 14$ repetitions with a water polo ball. Both groups trained eight weeks twice per week in addition to their regular water polo training. Throwing velocity was measured with a Doppler radar gun before and after the training period. Testing included throws with a water polo ball on land and in water, as well as with 1-kg and 3-kg medicine balls on land. Statistically significant increases were found in mean peak throwing velocity with the water polo, 1-kg, and 3-kg medicine balls after training. No differences between the groups were found, except in throwing velocity with water polo on land, with a statistically significantly larger increase for the combination training group $(+7.6 \%)$ than the medicine-ball training group $(+3.4 \%)$. These findings indicate that after training with the same workload (impulse), increases in throwing velocity in water polo are similar and suggesting workload may be a critical variable for training results.
\end{abstract}

Shooting on goal is very important in water polo (Smith, 1998) because it determines the success of every attack (Vila, Ferragut, Argudo, Abraldes, Rodríguez, \& Alacid, 2009). The water polo shot is a skill in which the players throw the ball as fast and accurately as possible to attempt scoring goals. A faster shot has a greater chance of scoring because

\footnotetext{
${ }^{1}$ Address correspondence to Daniel A. Marinho, University of Beira Interior, Sports Science Department, Rua Marquês d'Ávila e Bolama, 6201-001 Covilhã, Portugal or e-mail (dmarinho @ubi.pt).

${ }^{2}$ The authors thank the players who participated in this study.
} 
the goalkeeper has less time to react to the ball (Vila, et al., 2009). Thus, throwing the ball with great velocity seems to be an important performance indicator for water polo players (Stirn \& Strojnik, 2006). Indeed, this shot is considered to be a powerful one (Clarys \& Lewillie, 1971) by elite male players achieving peak ball velocities higher than $20 \mathrm{~m} \cdot \mathrm{sec}^{-1}$ (Feltner \& Taylor, 1997; Darras, 1999; Stirn \& Strojnik, 2006).

Different training strategies have been proposed to enhance throwing performance. In sports such as baseball, cricket, and handball, training programs based on the principles of overload and specificity (van den Tillaar, 2004; Grassi, Turci, Shirai, \& Sforza, 2006; Marques, Marinho, \& van den Tillaar, 2010) merely reported a positive effect on throwing velocity. Training with added resistance also positively influenced throwing velocity (Wooden, Greenfield, Johanson, Litzelman, Mundrane, \& Donatelli, 1992; Adams, Bangerter, \& Roundy, 1998). Although there have been several studies on training with medicine balls (e.g., Newton \& McEvoy, 1994; van den Tillaar, 2004; Szymanski, Bradford, Schade, \& Pascoe, 2007; Ettema, Gløsen, \& van den Tillaar, 2008; van den Tillaar \& Marques, 2009; van den Tillaar \& Marques, 2011), most did not control the workload between training groups (Newton \& McEvoy, 1994; Szymanski, et al., 2007). At present only three studies (Ettema, et al., 2008; van den Tillaar \& Marques, 2009; van den Tillaar \& Marques, 2011) have investigated the effects of different throwing training programs with the same workload in throwing velocity. However, none tested experienced water polo players. Further, none of these studies combined heavy medicine ball throwing with regular ball throwing as a part of specific strength training.

In this study, effects of two different throwing training programs with the same workload (i.e., the same mechanical linear impulse) on throwing velocity with a water polo ball on land and in water, as well as with medicine balls of 1 and $3 \mathrm{~kg}$ on land were examined. It was hypothesized that throwing velocity would increase given the increased workload, but there would be no differences between the two groups since the workload was the same. Eventual differences in throwing velocity after training would be the result of specificity of the training programs.

\section{MetHOD}

\section{Participants}

Thirty experienced male water polo players took part in this study $(M$ age $=17.1$ yr., $S D=4.9 ; M$ body mass $=71.2 \mathrm{~kg}, S D=14.7 ; M$ height $=1.75$ $\mathrm{m}, S D=0.09 \mathrm{~m}$ ). All participants were junior category athletes with more than four years of previous training. Participants were included if they were under 18 years old and above 16 years of age, free from injury, and trained regularly for at least six times per week. Athletes with any type of chronic disease or with an orthopedic limitation were excluded. 
Participants were fully informed about the protocol before participating. Informed consent was obtained prior to testing from participants as well as from their parents, in accordance with the recommendations of the local ethical committee and current ethical standards in sports and exercise research.

\section{Experimental Design}

The study was conducted using two groups of junior amateur water polo players, matched on throwing velocity. Each group followed a different training program. One group only threw medicine balls, whereas the other group trained by throwing a combination of water polo balls plus medicine balls. Both groups had the same training workload (i.e., total impulse). The training workload was calculated by using the net impulse applied to the ball from initiation to ball release generated per throw, the same methods used by Ettema, et al. (2008) and van den Tillaar and Marques $(2009,2011)$. Net impulse $\left(\int F d t\right)$ was considered a highly relevant measure for resistance training, as it measures the total amount of force applied to the ball during the throwing movement. In ball throwing, momentum of the ball at release $\left(m v_{\text {rel }}\right)$ was used to indicate net impulse, since initial momentum was equal to zero $\left(\int F d t=\mathrm{D} m v=m v_{\text {rel }}\right)$. Therefore, the training programs were based upon either high resistance (medicineball training group) or a combination of high resistance and velocity (combination training group). Two-handed overhead ball throws were used for training, as previously done by van den Tillaar and Marques (2009). According to these authors, this type of overhead throw will possibly limit the degrees of freedom, as there is only a small rotation along the longitudinal axis. This makes the performance less dependent on differences in individual technique among participants. Data so generated can be compared for effects of different training regimens. Given that the present aim was to compare effects of specific strength training (medicine-ball training) vs combination training and not the absolute effect of training, no control group was included.

\section{Testing Procedures}

Throwing velocity was tested before and after training, with a water polo ball, and 1-kg and 3-kg medicine balls. Before the pre-test, the participants were familiarized by throwing with different weighted balls in separate training sessions. These activities were undertaken to avoid a learning effect. Pre- and post-tests were performed by measuring peak throwing velocity with a water polo ball (circumference $0.68 \mathrm{~m}$ and mass $0.45 \mathrm{~kg}$ ) on land and in water, a 1-kg medicine ball with a circumference of $0.72 \mathrm{~m}$ and a 3-kg medicine ball with a circumference of $0.78 \mathrm{~m}$. A general upper-body warm-up of $10 \mathrm{~min}$., which included throwing 
with the differently weighted balls, preceded testing. The same testing procedure reported by van den Tillaar and Marques (2009) was applied. For the medicine ball throwing, the participants placed both feet parallel to throw the balls. In this starting position, participants held the ball before their body with both hands. The participants were instructed to throw the medicine ball as far and fast as possible forwards over their head (as in soccer throw-in movement technique). Both feet were held on the ground at all times during and after the throw. Torso and hip rotation were not allowed. When a participant did not follow the rules during the throw the attempt was discarded and a new attempt was performed. The research leader, who had years of experience in throwing, controlled this aspect of the study. Five valid attempts with each ball were measured with $1 \frac{1}{2} \mathrm{~min}$. of rest between attempts. Participants were also required to throw a water polo ball on land with maximal velocity. An overhead shot was performed as they did in the water. Standing with two feet firmly on the ground during and after the throw was required. No preliminary steps were permitted.

The throwing sequence with the three different balls was randomized for each participant so fatigue and learning would not alter performance. Peak velocity was determined by a Doppler radar gun (Sports Radar 3300, Sports Electronics, Inc.), with $\pm 0.03 \mathrm{~m} \cdot \mathrm{sec}^{-1}$ accuracy within a field of $10^{\circ}$ from the gun. The radar gun was positioned $1 \mathrm{~m}$ behind the participant at ball height during the throw. The peak velocity for the three best attempts was used for further analysis.

Following the land throws with medicine balls and the water polo ball, participants performed water polo shots to a goal from the penalty line ( $5 \mathrm{~m}$ from the goalie) with their dominant arm. Peak ball velocity was measured from behind the goalie. The ball was not allowed to touch the water or hit the goal posts. Five valid trials were recorded and the mean peak velocity was used for further analysis.

After the initial test, participants were matched on their throwing velocity with the water polo ball in the water and assigned to either a medicine ball training group $(n=14)$ or a combination of medicine ball plus water polo ball training group (combination training group, $n=16$ ). All participants were required to use the same throwing technique: standing with both feet on the ground while throwing the ball (either a water polo or medicine ball) forwards over their heads (overhead throwing) as fast as possible against the wall located at 3 to $5 \mathrm{~m}$ distance (depending on their throwing skills). Comparison of pre-test scores for throwing with the 3-kg medicine ball and with regular weighted water polo ball showed the medicine ball throw resulted in an impulse of $8.5 \mathrm{Ns}$ per throw versus $1.78 \mathrm{Ns}$ when throwing with water polo balls. One training session for the strength- 
training group was three series of six repetitions with the medicine balls, which is a total workload of 153 Ns. To conduct the same workload in the combination training group the half of the workload was thrown with the medicine ball (9 times). The other $76.5 \mathrm{Ns}$ of workload were throws with the water polo ball (1.78 Ns per throw). Thus, the combination training group had to throw 43 times with the water polo ball and nine times with the medicine ball to match the workload of the medicine ball training group. To avoid fatigue, the medicine ball training group performed $3 \times 6$ repetitions with a $3-\mathrm{kg}$ medicine ball, whereas the combination training group completed $1 \times 9$ repetitions with the $3-\mathrm{kg}$ ball followed by $3 \times 14$ repetitions with a water polo ball, always with 2 -min. recoveries between the sets. Each participant performed these exercises twice per week for eight consecutive weeks, in addition to their normal water polo training. The participants did not undertake any other type of resistance training during the present study. An expert coach (one of the authors) supervised each training session to ensure all participants threw with correct technique and followed the experimental protocol design.

\section{Data Analysis}

Comparison of the effects of the training protocols, a mixed design 2 (Test Occasion: pre-test, post-test repeated measures) $\times 2$ (group: medicine ball, combination training) analysis of variance (ANOVA) for each ball was performed. Anthropometric measurements of the training groups were compared by performing a $t$ test for each variable. Statistical significance was set at $p<.05$. Data were expressed as mean, and percentage of change from the pre- to post-test was calculated to compare the results with those of other studies. Effect size was evaluated with partial $\eta^{2}$ (partial eta squared) where 0.01 constitutes a small effect, 0.06 a medium effect, and 0.14 a large effect (Cohen, 1988).

\section{RESULTS}

No statistically significant differences $(p \geq .32)$ in anthropometric measurements were found between the groups at pretest. A statistically significant main effect of test occasion (pre- and post-test) was found for ball velocity of every kind of ball $\left(F \geq 11.2, p \leq .002\right.$, partial $\left.\eta^{2} \geq 0.28\right)$ (Table 1), i.e., both groups increased in mean peak ball-throwing velocity with each ball type after training. However, only an interaction effect was found between groups in throwing velocity with the water polo ball on land ( $F=4.8, p=.004, \eta^{2} \geq 0.15$; Table 1), i.e., the combination-training group showed a greater magnitude of increase $(7.6 \%)$ than the medicine ball training group (3.4\%) in throwing velocity with the water polo ball. With the other balls, no statistically significant differences between groups were found $\left(F \leq 2.4, p \geq .01, \eta^{2} \leq 0.08\right)$. 
TABLE 1

Descriptive Statistics for Peak Throwing Velocity $\left(\mathrm{M} \cdot \mathrm{SEC}^{-1}{ }^{-1}\right)$ With a Standard Water Polo Ball in the Water, on Land, and With Medicine Balls at 1 and 3 Kg FOr Both Groups at Pre- AND Post-test

\begin{tabular}{|c|c|c|c|c|c|c|c|c|}
\hline \multirow[t]{3}{*}{ Group } & \multicolumn{4}{|c|}{ Medicine Ball Training } & \multicolumn{4}{|c|}{ Combination Training } \\
\hline & \multicolumn{2}{|c|}{ Pre-test } & \multicolumn{2}{|c|}{ Post-test } & \multicolumn{2}{|c|}{ Pre-test } & \multicolumn{2}{|c|}{ Post-test } \\
\hline & $M$ & $S D$ & $M$ & $S D$ & $M$ & $S D$ & $M$ & $S D$ \\
\hline Water polo in water & 14.66 & 1.81 & 15.04 & 1.72 & 14.71 & 1.82 & 15.06 & 1.78 \\
\hline Water polo on land & 15.87 & 1.38 & $16.41^{*}$ & 1.65 & 14.79 & 1.65 & $15.91^{*}$ & 1.90 \\
\hline 1-kg medicine ball & 9.96 & 1.46 & 10.56 & 1.22 & 9.60 & 1.53 & 10.53 & 0.92 \\
\hline 3-kg medicine ball & 7.10 & 0.96 & 7.98 & 1.03 & $6.94^{*}$ & 0.97 & 7.50 & 0.74 \\
\hline
\end{tabular}

Note.-The peak throwing velocity increased from pre- to post-test in every group and with every ball $(p<.05)$. *Significant difference in magnitude of increase between the two groups from pre- to post-test $(p=.04)$.

\section{DisCUSSION}

The present study was designed to elaborate the effects of two throwing programs with the same workload on mean peak throwing velocity with a water polo ball on land and in water as well as with medicine balls of 1 and 3-kg. Both training programs lead to statistically significant increases in throwing velocity with all ball types (water polo ball on land and in water as well as 1- and 3-kg medicine ball on land) after the eight weeks of training. The combination training group obtained a statistically significantly greater increase in mean peak throwing velocity with the water polo ball on land than the medicine ball training group.

Although some studies have examined the effect of different training programs on throwing velocity, none was conducted with water polo players or used a workload control. The studies performed by Ettema, et al. (2008) and van den Tillaar and Marques $(2009,2011)$ used similar training programs with the same workload and yielded data in line with current findings. Ettema, et al. (2008) reported statistically significant increases in throwing velocity $(3.6 \%)$ with regular weighted balls after eight weeks training of two groups (velocity training: $6.1 \%$ and resistance training: $1.4 \%$ ) of experienced female handball players. Also, van den Tillaar and Marques (2011) found a statistically significant increase in throwing velocity with a soccer ball (6.9\%) after three different training programs (resistance training, combination training, and velocity training). These authors, in a previous study (van den Tillaar \& Marques, 2009), compared the effects of two distinct training programs (velocity training and resistance training) in university students on throwing velocity and distance with a soccer ball and found similar increases of $4.2 \%$. The results of these studies (Ettema, et al., 2008; van den Tillaar \& Marques, 2009, 2011) showed a statistically significant improvement in throwing velocity with the regular weighted balls 
(handball and soccer ball) after a short (six to eight weeks) training varying from 1.4 to $6.9 \%$.

However, van den Tillaar and Marques (2011) also reported that after six weeks of training, throwing velocity with a 3-kg medicine ball did not change and even decreased in one group. The authors justified these results by the short duration and relatively low volume of training. A sixweek training does not seem long enough to increase throwing velocity with heavier balls. It is possible that a longer training of eight or 10 weeks or higher training volume would yield different results. Eight weeks of training seems to be enough to statistically significantly increase throwing velocity with heavier balls in the medicine ball training and combination training groups. In fact, the training volume may be a key in improving throwing (van den Tillaar \& Marques, 2009). It may not be problem to duplicate the volume in the medicine ball training group without the quality of throwing being affected by fatigue and might positively change throwing performance even more.

When comparing the results between groups, an interaction effect was found in throwing velocity with the water polo ball on land, i.e., the combination training group had a higher increase $(7.6 \%)$ than the medicine ball training group (3.4\%). In contrast, van den Tillaar and Marques (2009) and Ettema, et al. (2008) did not detect such statistically significant differences between their groups, and concluded that both training forms (resistance or velocity) may yield similar effects. However, in these studies the velocity training group (throwing with soccer balls) increased throwing velocity more than the resistance training group, emphasizing the importance of training specificity. Also, van den Tillaar and Ettema (2009) found differences in ball velocity and throwing accuracy when comparing dominant and non dominant arm of team handball players, which could be an interesting issue to consider, since only throws by the dominant arm were analyzed during the water polo throwing.

In summary, these findings indicate that after training with the same workload (impulse) increases in throwing velocity in water polo are similar and suggesting workload may be a critical variable for training results. Present findings also strengthen the importance of training specificity since the combination training group followed a type of training that is more specific to water polo ball throwing on land.

\section{REFERENCES}

AdAms, T. B., BAngerter, B. L., \& Roundy, E. S. (1998) Effect of toe and wrist/finger flexor strength training on athletic performance. Journal of Applied Sport Science Research, 2, 3-34.

Clarys, J., \& Lewillie, L. (1971) The description of wrist and shoulder motion of different waterpolo shots using a simple ligthtrace technique. In L. Lewillie \& J. Clarys 
(Eds.), Biomechanics in swimming I: Proceedings of the 1st International Symposium of Biomechanics in Swimming. Brussels: Presse Universitaire Bruxelles. Pp. 249-255.

CoHen, J. (1988) Statistical power analysis for the behavioral sciences. (2nd ed.) Hillsdale, NJ: Erlbaum.

DARRAS, N. G. (1999) Maximum shooting velocity in waterpolo direct shot and shot with faints of the international level athletes participating in the 10th FINA World Cup. In K. Keskinen, P. Komi, \& A. P. Hollander (Eds.), Biomechanics and medicine in swimming VIII: Proceedings of the 8th International Symposium on Biomechanics and Medicine in Swimming. Jyväskylä, Finland: Univer. of Jyväskylä . Pp. 185-190.

EtTema, G., Gløsen, T., \& VAN DEN TillaAr, R. (2008) Effect of specific strength training on overarm throwing performance. International Journal of Sports Physiology and Performance, 3, 164-175.

Feltner, M., \& TAYLOR, G. (1997) Three-dimensional kinetics of the shoulder, elbow, and wrist during a penalty throw in water polo. Journal of Applied Biomechanics, 13, 347-372.

Grassi, G. P., Turci, M., ShiRAI, Y. F., \& Sforza, C. (2006) Two ball-throwing tasks. Perceptual and Motor Skills, 102, 19-28.

Marques, M. C., Marinho, D. A., \& van den Tillaar, R. (2010) A load-velocity relationship for men and women in overhead throwing performance. Journal of Sports Science and Medicine, 9, 524-526.

Newton, R. U., \& McEvor, P. (1994) Baseball throwing velocity: a comparison of medicine-ball training and weight training. Journal of Strength and Conditioning Research, 8, 198-203.

Sмiтh, H. K. (1998) Applied physiology of water polo. Sports Medicine, 26, 317-334.

Stirn, I., \& StrojniK, V. (2006) Throwing with different kinetic chains. Portuguese Journal of Sport Sciences, 6(Suppl. 2), 98-100.

Szymanski, D. J., Szymanski, J. M., Bradford, T. J., Schade, R. L., \& Pascoe, D. D. (2007) Effect of twelve weeks of medicine-ball training on high school baseball players. Journal of Strength and Conditioning Research, 21, 894-901.

VAN DEN TILLAAR, R. (2004) Effect of different training programs on the velocity of overarm throwing: a brief review. Journal of Strength and Conditioning Research, 18, 388-396.

van den Tillaar, R., \& Ettema, G. (2009) A comparison of overarm throwing with the dominant and nondominant arm in experienced team handball players. Perceptual and Motor Skills, 109, 315-326.

van Den TillaAR, R., \& Marques, M. C. (2009) Effect of two different throwing training programs with same workload on throwing performance with soccer ball. International Journal of Sports Physiology and Performance, 4, 474-484.

VAN Den TIllaAar, R., \& MARQues, M. C. (2011) A comparison of three training programs with same workload on overhead throwing velocity. Journal of Strength and Conditioning Research, 25, 2316-2321.

-Vila, H., Ferragut, C., Argudo, F. M., Abraldes, J. A., Rodríguez, N., \& Alacid, F. (2009) Relationship between anthropometric parameters and throwing velocity in water polo players. Journal of Human Sport and Exercise, 4, 57-68.

Wooden, M. J., Greenfield, B., Johanson, M., Litzelman, L., Mundrane, M., \& DonatelLI, R. A. (1992) Effects of strength training on throwing velocity and shoulder muscle performance in teenage baseball players. Journal of Orthopaedic and Sports Physical Therapy, 1, 223-228.

Accepted November 9, 2012. 